\title{
Directional Wetting in Anisotropic Inverse Opals
}

\section{Citation}

Phillips, Katherine R., Nicolas Vogel, Ian B. Burgess, Carole C. Perry, and Joanna Aizenberg. 2014. "Directional Wetting in Anisotropic Inverse Opals." Langmuir 30 (25) (June 18): 7615-7620. doi:10.1021/la5015253.

\section{Published Version}

doi:10.1021/la5015253

\section{Permanent link}

http://nrs.harvard.edu/urn-3:HUL.InstRepos:37255329

\section{Terms of Use}

This article was downloaded from Harvard University's DASH repository, and is made available under the terms and conditions applicable to Open Access Policy Articles, as set forth at http:// nrs.harvard.edu/urn-3:HUL.InstRepos:dash.current.terms-of-use\#OAP

\section{Share Your Story}

The Harvard community has made this article openly available.

Please share how this access benefits you. Submit a story.

Accessibility 


\title{
Directional Wetting in Anisotropic Inverse Opals
}

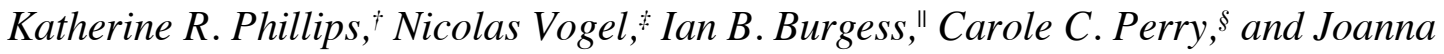

Aizenberg ${ }^{\dagger, \vec{*}, \|, *}$

'Department of Chemistry and Chemical Biology, ${ }^{\circledR}$ School of Engineering and Applied Sciences, and "Wyss Institute for Biologically Inspired Engineering, Harvard University, Cambridge, MA 02138, U.S.A.; and ${ }^{\S}$ Interdisciplinary Biomedical Research Centre, Nottingham Trent University, Clifton Lane, Nottingham NG11 8NS, U.K.

\section{AUTHOR INFORMATION}

\section{Corresponding Author}

*Correspondence should be addressed to jaiz@seas.harvard.edu

\begin{abstract}
Porous materials display interesting transport phenomena due to the restricted motion of fluids within the nano- to micro-scale voids. Here, we investigate how liquid wetting in highly ordered inverse opals is affected by anisotropy in pore geometry. We compare samples with different degrees of pore asphericity and find different wetting patterns depending on the pore shape. Highly anisotropic structures are infiltrated more easily than their isotropic counterparts. Further, the wetting of anisotropic inverse opals is directional, with liquids filling from the side more easily. This effect is supported by percolation simulations as well as direct observations of wetting using time-resolved optical microscopy.
\end{abstract}


KEYWORDS. porous materials, wetting, photonic crystals, transport phenomena, inverse opals, anisotropy.

\section{Introduction}

The porous structure known as an inverse opal is formed by backfilling a three-dimensional sacrificial template of colloidal particles. This highly ordered structure acts as a photonic crystal, strongly interacting with electromagnetic radiation with wavelength on the same length scale as the structural periodicity. When this length scale corresponds to visible light, inverse opals display bright, iridescent colors. ${ }^{1-5}$ While the color originates from the periodic nature of the inverse opal's regular pore structure, the pores are also interconnected through small openings referred to as necks. This porosity gives inverse opals a high accessible surface area, making them useful as catalyst supports ${ }^{6,7}$ and electrodes. ${ }^{8-10}$ Additionally, their interconnected, regular pore structure provides a model system to study the behavior of fluids within nanoscale porous materials. ${ }^{11,12}$

We recently developed a co-assembly method to simultaneously crystallize the sacrificial template and the surrounding silica matrix of an inverse opal in a convective process. ${ }^{13}$ This method creates an inverse opal structure with a single crystal orientation, few cracks, high order, and a narrow distribution of neck sizes, all over large $(\mathrm{cm})$ length scales, forming an ideal model system to study liquid wetting in the confinement of nanoscale porous media. When liquids infiltrate the pores, the structure loses its color due to the lower refractive index contrast between the silica matrix and the liquid as compared to the matrix and air. ${ }^{14,15}$ The high degree of order obtainable with co-assembly and the narrow distribution of neck sizes separating the individual pores lead to a sharp transition from wetting to nonwetting. The wetting event can be visualized 
by simple observation of the color change, ${ }^{14}$ much like the color change for Morpho butterfly wings in different solvents..$^{16,17}$

The surface chemistry and specific pore geometry of inverse opals determine whether or not a given liquid will fill the structure spontaneously upon contact. Using alkylchlorosilanes, ${ }^{18}$ silica inverse opals can be chemically functionalized to prevent or promote liquid infiltration into the pores of the inverse opal. ${ }^{14,19}$ Lateral patterning with different alkylchlorosilanes enables spatially confined wetting, allowing the design of colorimetric sensors that are sensitive to the surface tension and chemical identity of the infiltrating liquid. ${ }^{20}$ While the effect of chemical functionalization of inverse opals on their wetting profiles has been assessed, the role of geometry, and more specifically how changes in the shape of the pores and necks alter inverse opals' wetting properties, has not been studied. The pore-to-pore connection through the neck in inverse opals exhibits re-entrant curvature, which is known to impede wetting..$^{21-23}$ This re-entrant curvature can be quantified by the "neck angle," the tangent angle that the necks form with the pore wall. We anticipated that anisotropic pore geometries lead to non-uniform neck angles that modify the isotropic wetting profiles of the opal structures.

We previously described the fabrication of anisotropic inverse opals using high calcination temperatures, where the elliptical pore geometry originates from condensation-induced shrinking perpendicular to the substrate. ${ }^{24}$ Here, we report that the introduction of anisotropy into the pore structure introduces directionality in the wetting behavior, affecting the structure's response to liquids on the macroscopic scale. We corroborated experimental observations with percolation modeling and show that anisotropy of the inverse opal pore geometry changes the microscopic liquid pinning at the necks thereby making the structures easier to wet macroscopically. For 
highly anisotropic samples, the difference can be such that percolation in the plane parallel to the substrate dominates the wetting behavior.

\section{Materials and Methods}

Experimental Details. The inverse opal synthesis is described in detail elsewhere. ${ }^{13}$ Briefly, it consists of the simultaneous assembly of the colloidal crystal template with the silica matrix. Polystyrene colloids were made using a surfactant-free emulsion polymerization process ${ }^{25}$ and were diluted with water to $0.1 \%$ solid content in $20 \mathrm{~mL}$ total volume for assembly. $150 \mu \mathrm{L}$ of pre-hydrolyzed tetraethyl orthosilicate (TEOS) solution $(1: 1.5: 1$ by weight of TEOS : ethanol : $0.1 \mathrm{M} \mathrm{HCl}$, stirred for $1 \mathrm{~h}$ ) was added and the mixture underwent sonication. A silicon wafer substrate was suspended vertically in the vial. The vial was then placed inside a Memmert UF110 oven for several days at $65^{\circ} \mathrm{C}$ to allow for evaporation of the solvent. The colloidal template was removed with tetrahydrofuran or by calcination at high temperatures. ${ }^{24}$ Here, four calcination temperatures were used in addition to solvent removal: $500^{\circ} \mathrm{C}, 800^{\circ} \mathrm{C}, 1000^{\circ} \mathrm{C}$, and $1100^{\circ} \mathrm{C}$, for a total of five degrees of anisotropy. Samples were cleaned in acid piranha, a 3:1 mixture of sulfuric acid (95-98\%, Sigma) and hydrogen peroxide (30\%, from Aqua Solutions), and then exposed to n-decyltrichlorosilane (Gelest) vapor under vacuum for $24 \mathrm{~h}$, as described elsewhere. ${ }^{20}$ Deionized water was used from a Milli-Q system, ethanol was obtained from Koptec, and all other reagents $(0.1 \mathrm{M} \mathrm{HCl}$, styrene, tetraethylorthosilicate, isopropanol) were obtained from Sigma Aldrich and used without further treatment.

Imaging of the inverse opal samples was performed using a Zeiss Ultra Plus field emission scanning electron microscope (SEM). The images were then analyzed in ImageJ using the pixel size from the Zeiss SEM software. Optical images were taken under diffuse lighting on a Canon 
EOS 40D with Macro lens. Intrinsic contact angles were measured on flat surfaces with matching surface functionality using a KSV Instruments system and KSV CAM2008 software. The time-resolved microscopy images were taken on a Leica DMRX microscope using Image Pro-Plus software.

Simulation Description. Neck angles for each pore geometry were calculated as described in the supplemental information. These angles were used as an input in a percolation simulation performed in MATLAB, with each pore of the three-dimensional crystal represented as an element in a three-dimensional matrix. The simulation was adapted from that described in reference 20, with modifications to account for pore anisotropy. In the simulation, each pore was connected to 12 nearest neighbors through 12 necks in an fcc lattice; each neck was characterized completely by its neck angle, the tangent angle to the pore wall at the end of the neck. For ideal elliptical pores, there are two distinct neck angles: the in-plane neck angle (made with adjacent pores in the same horizontal plane parallel to the substrate), and the out-of-plane neck angle (made with adjacent pores in the horizontal plane above or below). Further, while the out-of-plane neck angle varies along the perimeter of the neck due to anisotropy, we used the maximum angle along this boundary as the out-of-plane neck angle.

To start, either the top layer or the side layer of pores was considered filled initially (i.e. the matrix element $=1$ ), with the rest of the pores left empty (i.e. the matrix element $=0$ ). Liquid infiltration is then described entirely by its contact angle (see main text for a discussion of this point). If the contact angle was smaller than the neck angle, that neck was "open" and a filled pore on one side of the neck filled an empty pore on the other. Conversely, if an empty pore's neck angles were all smaller than the infiltrating liquid's contact angle, the pore was left empty regardless of the filling of its neighbors. The computer then iterated through each pore in the 
matrix, filled any pores that could be filled, and continued until no further changes were observed. The fraction of pores filled was recorded for each liquid.

\section{Results and Discussion}

Both the identity of the liquid and the geometry of the structure determine whether or not a liquid will infiltrate an inverse opal. In particular, the surface tension of a liquid and its liquidsurface interaction influence the wetting behavior. ${ }^{20}$ The intrinsic contact angle $\left(\theta_{C}\right.$, defined by Young's equation ${ }^{26}$ ) is a useful parameterization of these surface energies for studying wetting behavior. An estimate for $\theta_{C}$ is the angle that a droplet of the liquid forms with a flat surface (Figure 1A). A low intrinsic contact angle indicates a favorable interaction with the surface, facilitating wetting.

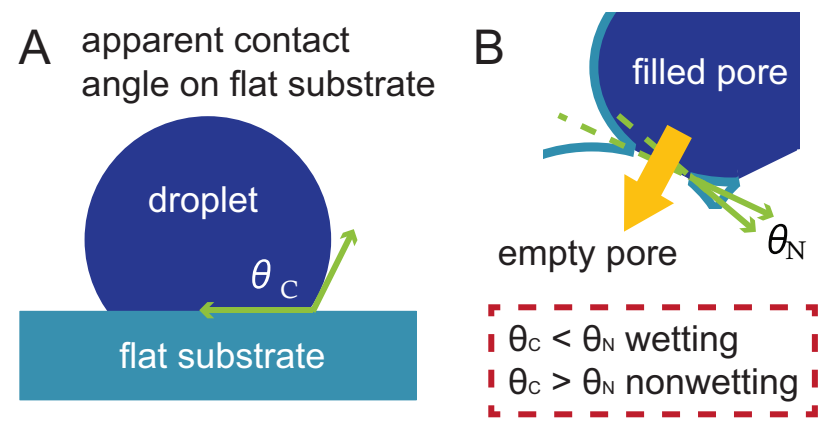

Figure 1. Theoretical conditions for wetting. (A) The intrinsic contact angle, $\theta_{C}$, is estimated by the angle that a droplet makes on a flat surface. (B) The neck angle, $\theta_{N}$, is the re-entrant angle for a liquid pinned at a neck, formed between the neck and the tangent line to the pore wall. In order to overcome this barrier and wet the structure, the contact angle must be smaller than the neck angle $\left(\theta_{C}<\theta_{N}\right)$.

The neck angle, the angle formed between the neck and the tangent line to the pore wall at the neck, is the key structural feature that determines wetting. ${ }^{20}$ It is the re-entrant angle that the liquid contact line "sees" as it emerges from a neck $\left(\theta_{N}\right.$ in Figure 1B). Even for inverse opals with lyophilic surface chemistry $\left(\theta_{C}<90^{\circ}\right)$, filling will be restricted as the liquid front emerges from the narrow necks. For the liquid to enter the pore, its intrinsic contact angle must be smaller 
than the neck angle. In this situation, a concave meniscus shape draws the liquid into the next pore. If the contact angle is larger than the neck angle, the liquid will show a convex-shaped meniscus at the neck, which prevents the liquid from entering, in analogy to re-entrant curvatures in omniphobic surface structures ${ }^{21-23}$ and pinning on conical frustrums and spheres on the mmlength scale..$^{27-29}$ Alternatively, this pinning can be understood by considering a free-energy tradeoff between the favorable wetting interaction of the pore walls and the cost of expanding the liquid-air interface. As a result, a liquid front can only move from one pore to the next when $\theta_{\mathrm{C}}$ is sufficiently low to overcome this tradeoff. Thus, we obtain $\theta_{\mathrm{C}}<\theta_{\mathrm{N}}$ as the condition for wetting, with $\theta_{\mathrm{C}}=\theta_{\mathrm{N}}$ as the cutoff. ${ }^{14,20}$ Based on this inequality, only the relative sizes of $\theta_{\mathrm{C}}$ and $\theta_{\mathrm{N}}$, and not the absolute sizes, influence the behavior. The barrier for entry increases with $\theta_{\mathrm{C}}$, as shown in Supplemental Figure S7, with 1.2 atm of pressure necessary for $\theta_{\mathrm{C}}=\theta_{\mathrm{N}}+1^{\circ}$ and 5.4 atm for $\theta_{\mathrm{C}}=\theta_{\mathrm{N}}+20^{\circ}$ (which is equal to submerging under $1.6 \mathrm{~m}$ and $46 \mathrm{~m}$ water, respectively).

Anisotropic pore geometry and neck angles. By varying the calcination temperature, inverse opal pore geometries with adjustable anisotropy can be realized. Representative scanning electron microscope (SEM) images of such samples are shown in Figure 2A-B. After coassembling the template and matrix, the isotropic inverse opal (Figure 2A) was made by dissolving the colloidal template with tetrahydrofuran, and the anisotropic inverse opal sample (Figure 2B) was prepared using an elevated calcination temperature of $1000^{\circ} \mathrm{C} \cdot{ }^{13,24}$ We quantify the degree of anisotropy in these samples using the ratio of the width to the height $(w: h)$ measured from SEM images. ${ }^{24}$

Anisotropic pores change the neck angles, designated by $\theta_{N}$ or $\theta^{\prime}{ }_{N}$ in Figure $2 \mathrm{C}$ and $\mathrm{D}$. We expect all neck angles to be equal (Figure 2C) in isotropic pores. As ellipticity is introduced into the system, the in-plane and out-of-plane neck angles $\left(\theta^{\prime}{ }_{N}\right.$ and $\theta_{N}$, respectively) begin to differ 
(Figure 2C-D), even though the actual neck sizes are kept constant. In particular, the angle between pores within a layer $\left(\theta^{\prime}{ }_{N}\right.$, Figure 2D) becomes larger than the angle between pores in different layers $\left(\theta_{N}\right)$.
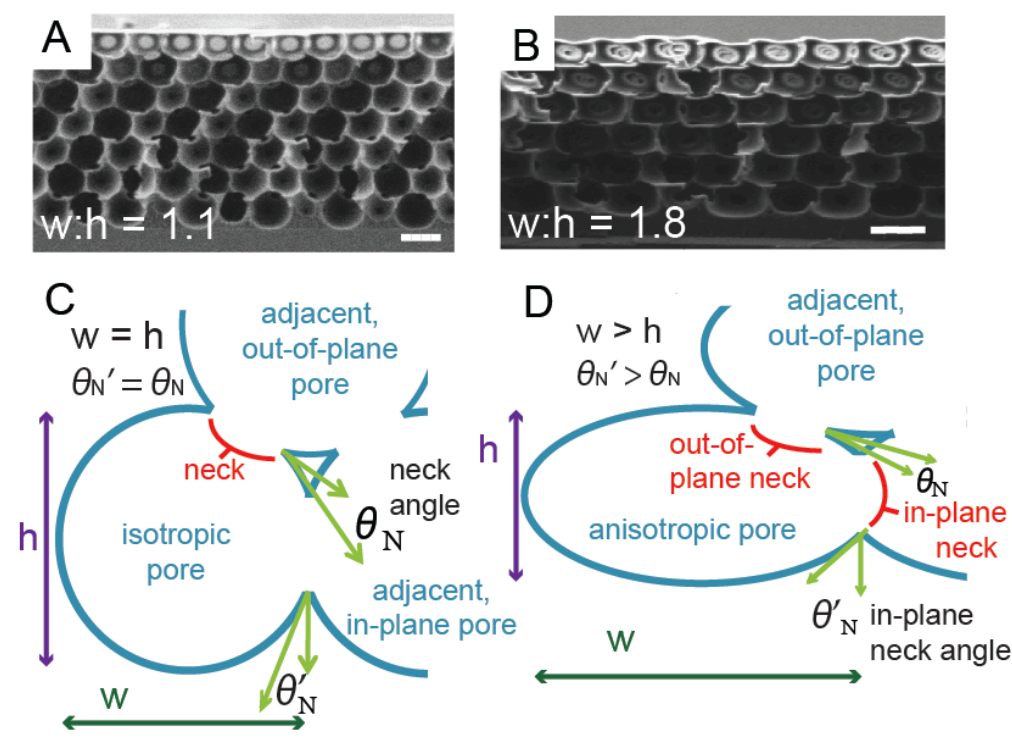

Figure 2. Pore structure of anisotropic inverse opals. (A-B) Scanning electron microscope images of isotropic and anisotropic inverse opals prepared by removing the colloidal template by (A) dissolving in tetrahydrofuran, and (B) calcination at $1000^{\circ} \mathrm{C}$, producing width to height ratios $(w: h)$ of 1.1 and 1.8 , respectively. Scale bars are $400 \mathrm{~nm}$. (C) In isotropic inverse opals, all necks have the same neck angle $\theta_{N}$. (D) With anisotropic pores, the in-plane (side) neck angle, $\theta^{\prime}{ }_{N}$, is larger than the out-of-plane (top) neck angle, $\theta_{N}$, for a constant neck size.

While a structure with spherical pores displays isotropic percolation as each pore is infiltrated with equal probability, anisotropic pores characterized by two different neck angles (in-plane and out-of-plane) are expected to have different wetting patterns in the two directions. Larger neck angles facilitate wetting, ${ }^{20}$ so anisotropy causes the in-plane and out-of-plane infiltration probabilities to become different. As $w: h$ increases, this effect is magnified: the out-of-plane neck angles continue to shrink, whereas the in-plane neck angles get larger (i.e. less re-entrant). Based on this difference in neck angles, we anticipated that the more anisotropic the pores, the easier it is for liquids to infiltrate them from the side, and the harder it is to infiltrate them from the top. 
Experimental colorimetric wetting. To experimentally determine the effect of anisotropy on the macroscopic wetting behavior, we prepared samples with a variety of $w: h$ ratios and tested their wettability in a range of liquids (Figure 3). All samples were made with $410 \mathrm{~nm}$ colloidal spheres as a template and underwent calcination at various temperatures to create different pore geometries. ${ }^{24}$ Piranha treatment was used for all samples to ensure the presence of surface $-\mathrm{OH}$ groups before exposing the samples to $\mathrm{n}$-decyltrichlorosilane vapors in a desiccator for $24 \mathrm{~h},{ }^{14}$ and functionalization was confirmed with X-ray photoelectron spectroscopy (Supplemental Figure S1). After functionalization, these samples were submerged in mixtures of water and isopropanol (IPA) with gradually increasing IPA content to achieve a range of contact angles. The intrinsic contact angles were estimated from contact angle measurements on a flat surface with the same surface treatment as the inverse opals, shown in Figure 3A and listed in Table S1.

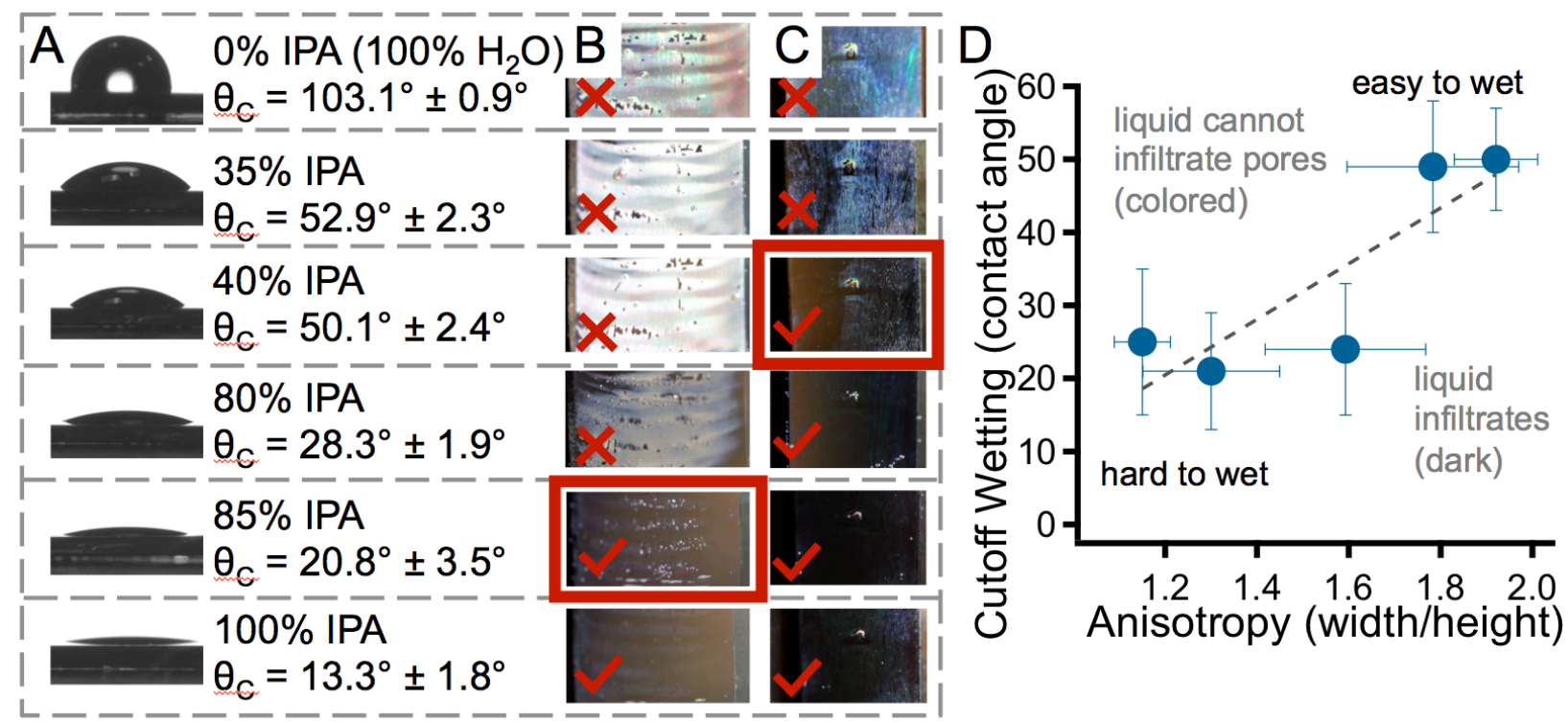

Figure 3. Effect of contact angle on the wetting characteristics of different inverse opals. (A) Contact angles of water and isopropanol (IPA) mixtures (v/v) on a flat silicon surface functionalized with $\mathrm{n}$ decyltrichlorosilane. (B-C) Photos of inverse opals made from $410 \mathrm{~nm}$ colloids as template, with the template removed by $(\mathrm{B})$ dissolution in tetrahydrofuran $(w: h=1.1)$ and $(\mathrm{C})$ calcination to $1100^{\circ} \mathrm{C}(w: h=$ 1.9), followed by functionalization with n-decyltrichlorosilane, and immersion in the same water and IPA mixtures. The wetting state (non-wetted/colored vs. wetted/filled) is indicated by an " $x$ " or " $\checkmark$ ", respectively. (D) Cutoff wetting as a function of anisotropy. The cutoff contact angle is defined as the 
contact angle on a flat surface of the water/IPA mixture that first wets the inverse opal film. Line is to guide the eye.

Indeed, we see a difference in the wetting of the samples with different anisotropy. Representative samples are shown in Figure 3B-C. The wetting is easily visible by the disappearance of color due to the loss of the refractive index contrast. Note that the starting color is different for the two samples due to the difference in periodicity originating from the anisotropy. ${ }^{24}$ The wetting state for the different inverse opals with the different water/IPA mixtures is indicated by crosses (" $x$ ”) for not-wetted/colored and checks (" $\checkmark$ ") for wetted/filled. Water does not wet any of the structures (top), and IPA wets all of them (bottom). For some intermediate mixtures, such as $80 \%(\mathrm{v} / \mathrm{v})$ IPA in water, wetting is observed only in the anisotropic structures (fourth row of Figure 3B-C). The cutoff wetting was determined as the highest contact angle (or, equivalently, the lowest IPA concentration, or, the highest surface tension liquid) that correlated with infiltration into the inverse opal film, as marked by the complete disappearance of color. Here, the cutoff for the left sample $(\mathrm{B}, w: h=1.1)$ occurs at $85 \%$ IPA, whereas the cutoff for the right sample $(\mathrm{C}, w: h=1.9)$ occurs at $40 \%$ IPA, as shown with the red boxes around these samples.

We quantified the effect of anisotropy on the wetting threshold of the inverse opals using liquids across a range of wetting abilities. All of the inverse opal films were tested in an array of $5 \%$ (vol) increments of IPA and water mixtures, each with a unique surface tension that falls in the range of 21 to $72 \mathrm{dyne} / \mathrm{cm},{ }^{30}$ resulting in different contact angles (Figure $3 \mathrm{~A}$ and Supplemental Table S1). Four samples were tested for each of the five different degrees of anisotropy. Figure 3D shows that the cutoff wetting depends on the pore geometry, generally getting easier to wet (wetting threshold occurring at higher $\theta_{C}$ ) with additional anisotropy. Since 
all the samples have been functionalized in the same manner, we attribute the observed trend to changes in the neck angle.

Wetting simulation. We simulated the wetting of a liquid with a given contact angle in isotropic and anisotropic inverse opals using a percolation model ${ }^{20}$ The simulation is described in detail in the methods; briefly, the next pore fills if it is connected to an adjacent, filled pore by a neck that allows liquid through by the criteria described above (i.e. if $\theta_{\mathrm{C}}<\theta_{\mathrm{N}}$ or $\theta_{\mathrm{C}}<\theta^{\text {, }}$, for out-of-plane and in-plane connections, respectively). The wetting threshold, or "cutoff wetting," is determined as the highest contact angle for a liquid that still fills $99.9 \%$ of the pores. At this concentration, unfilled pores are isolated scattering centers with an area density of $1 \%$ (assuming ten layers) or one per $\sim 10 \mu \mathrm{m}^{2},{ }^{20}$ insufficient to produce significant reflectance. ${ }^{31}$

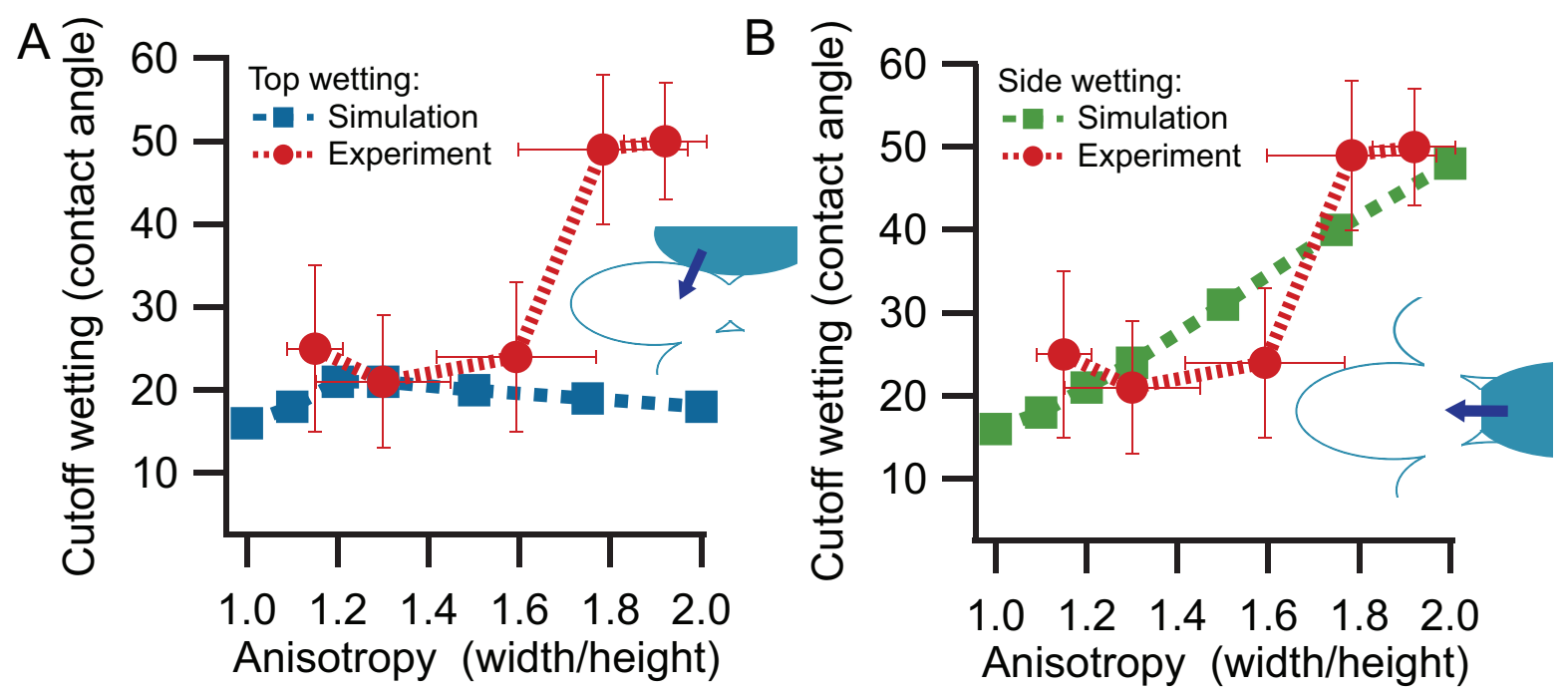

Figure 4. Simulated results of the wetting properties of the anisotropic inverse opals compared to experimental results, starting by filling (A) the top pores and (B) the side pores. "Top wetting" and "side wetting" are shown schematically in insets. Lines are to guide the eye.

We used the wetting simulation to model whether or not a liquid with a given contact angle will wick into a structure with a given neck angle in experimentally relevant conditions (Figure 4). The range of contact angles used in the simulation matched the range seen in Figure 3D. The 
neck angle input was calculated by approximating the pore structure as an ellipse and estimating the average neck size as half the horizontal pore radius. From this geometry, we calculated both in-plane and out-of-plane neck angles for each structure (see calculations in supplemental information) and assumed 10\% variance. These neck sizes were estimated based on SEM images of the structures such as those shown in Figure 2A-B.

The simulation clearly shows that wetting depends on anisotropy. The cutoff wetting was recorded for seven different $w: h$ ratios between 1.0 and 2.0. For isotropic pores $(1<w: h<1.2)$, the cutoff wetting was independent of the starting conditions. However, for anisotropic pores, the final wetting state of liquid depended on whether the liquid originated from the top (Figure 4A) or the side (Figure 4B) of the structure, with a strong dependence for highly elliptical pore shapes $(w: h>1.6)$. A liquid sitting on the top of the anisotropic pores is less able to wet the porous network, whereas wetting from the side is easier, because $\theta{ }_{\mathrm{N}}$ becomes increasingly larger than $\theta_{\mathrm{N}}$ with increasing anisotropy.

The experimental trend shown in Figure 3D most closely matches the simulation when the liquid originates from the side (Figure 4B), suggesting that liquids are filling from the side in anisotropic inverse opals. Since the in-plane neck angles are larger $\left(\theta^{\prime}{ }_{N}>\theta_{N}\right)$, and larger neck angles make it easier for liquids to fill, we conclude that wetting from the side causes the increase in wettability for highly anisotropic inverse opals. This increase in wettability implies that the liquid infiltration occurs directionally in the plane of the film. Besides the natural boundaries of the inverse opals, we expect that cracks in the structure might contribute to the wetting profile seen in our experimental results, as they allow liquid to access the pores from the side by penetration into the crack. In contrast, wetting of an isotropic structure is not as sensitive 
to cracks because its in-plane and out-of-plane neck angles are the same, leading to similar cutoffs for top and side wetting.

Directional wetting. Evidence for directional wetting was also confirmed experimentally. We used time-resolved optical imaging to observe liquid infiltration in the vicinity of a crack for both isotropic $\left(w: h=1.3 ; 500^{\circ} \mathrm{C}\right.$ calcination temperature $)$ and anisotropic $\left(w: h=1.8 ; 1000^{\circ} \mathrm{C}\right.$ calcination temperature) structures (Figure 5). In these experiments, a droplet was placed in the field of view of an optical microscope focused on an inverse opal, and pictures were taken (5 frames per second) to monitor the liquid's movement. The resulting video can be found in the supplemental information, with selected frames shown in Figure 5.

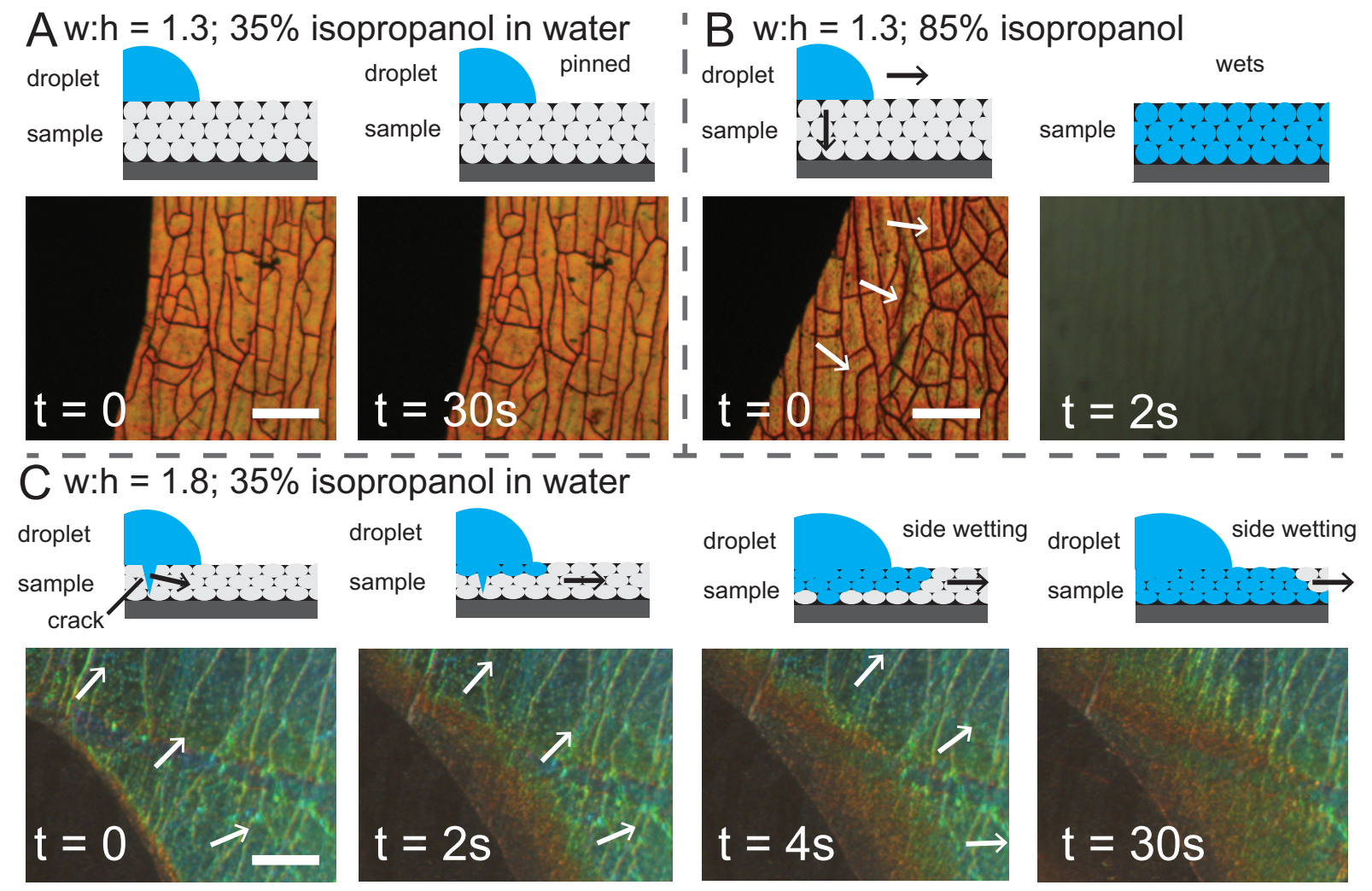

Figure 5. Optical images of isotropic and anisotropic wetting phenomena, with arrows to indicate liquid motion. (A-B) Isotropic ( $w: h=1.3$ ) inverse opal wetting in (A) $35 \%$ isopropanol (IPA) in water, and (B) $85 \%$ IPA in water. (C) Anisotropic wetting in an anisotropic inverse opal $(w: h=1.8)$ with 35\% IPA in water. Schematics of the phenomenon are shown above the optical images. Scale bars are $100 \mu \mathrm{m}$. 
In the sample with isotropic pores $(w: h=1.3)$, liquids either sit on top without filling the pores (low isopropanol concentration; Figure 5A), or they fill the entire structure (high isopropanol concentration; Figure 5B). The dark area represents the droplet sitting on top of the structure, as shown in the schematic, because light reflected from underneath the droplet is not captured by the microscope objective due to scattering off the top surface of the droplet. On a highly anisotropic sample $(w: h=1.8$, Figure $5 \mathrm{C})$, the liquid fills in from the side, allowing a clear view of the directional wetting. In this film with anisotropic pores, the color changes gradually, implying that individual layers of the structure may be filling. ${ }^{20}$ This behavior provides experimental evidence that the liquid infiltrates from the side of the structure with anisotropic pores, which we attribute to facilitated wetting due to larger neck angles. These results match well with the simulated wetting results based on theoretical neck angle calculations.

\section{Conclusions}

We have demonstrated how anisotropic pore geometries lead to directional wetting of a liquid through the three-dimensional porous network of an inverse opal. Elliptical pore geometries create different in-plane and out-of-plane inter-pore neck angles and sizes, causing directional wetting characteristics. Structures with very anisotropic pores are increasingly easier to wet, and we demonstrated with both simulations and time-resolved microscopy that in-plane wetting drives this trend.

Adjusting the geometry provides a convenient handle for controlling which liquids infiltrate inverse opals. Due to their order and porosity, inverse opals can be used to understand many properties of materials at the nanoscale. In particular, we expect these results to be of relevance for the general understanding of fluid dynamics in confined geometries as well as for the design of improved colorimetric sensors capable of differentiating liquids by surface tensions. The 
observation of a state that allows wetting from the side may also provide a convenient detection mechanism for cracks in photonic crystals.

\section{ASSOCIATED CONTENT}

Supporting Information. X-ray photoelectron spectroscopy confirming functionalization; contact angles of isopropanol and water mixtures; neck angle calculations for simulation; estimation of barrier to liquid filling; video of directional wetting. This material is available free of charge via the Internet at http://pubs.acs.org.

\section{AUTHOR INFORMATION}

\section{Corresponding Author}

*Email: jaiz@seas.harvard.edu

\section{Notes}

The authors declare no competing financial interests.

\section{ACKNOWLEDGMENT}

K.R.P. acknowledges support from a National Science Foundation Graduate Research

Fellowship and a National Defense Science and Engineering Graduate fellowship from the Department of Defense. N.V. acknowledges funding from the Leopoldina Fellowship Program. C.C.P. acknowledges the support of an Edward, Frances and Shirley B. Daniels and Wyss Fellowship while at the Radcliffe Institute for Advanced Study, 2012-2013. The authors thank A.V. Shneidman for helpful discussions. This work was funded with support from the Air Force 
Office of Scientific Research (AFOSR) under award number FA9550-09-0669-DOD35CAP, and it was performed in part at the Center for Nanoscale Systems (CNS) at Harvard University.

\section{REFERENCES}

(1) Sanders, J. Colour of Precious Opal. Nature 1964, 204, 1151-1153.

(2) Aguirre, C. I.; Reguera, E.; Stein, A. Tunable Colors in Opals and Inverse Opal Photonic Crystals. Adv. Funct. Mater. 2010, 20, 2565-2578.

(3) Schroden, R. C.; Al-Daous, M.; Blanford, C. F.; Stein, A. Optical Properties of Inverse Opal Photonic Crystals. Chem. Mater. 2002, 14, 3305-3315.

(4) Arsenault, A. C.; et al. From Colour Fingerprinting to the Control of Photoluminescence in Elastic Photonic Crystals. Nat Mater 2006, 5, 179-184.

(5) Freymann, von, G.; Kitaev, V.; Lotsch, B. V.; Ozin, G. A. Bottom-Up Assembly of Photonic Crystals. Chem. Soc. Rev. 2013, 42, 2528-2554.

(6) Chen, J. I. L.; Loso, E.; Ebrahim, N.; Ozin, G. A. Synergy of Slow Photon and Chemically Amplified Photochemistry in Platinum Nanocluster-Loaded Inverse Titania Opals. $J$. Am. Chem. Soc. 2008, 130, 5420-5421.

(7) Waterhouse, G. I. N.; Wahab, A. K.; Al-Oufi, M.; Jovic, V.; Anjum, D. H.; SunWaterhouse, D.; Llorca, J.; Idriss, H. Hydrogen Production by Tuning the Photonic Band Gap with the Electronic Band Gap of $\mathrm{TiO}_{2}$. Sci. Rep. 2013, 3 . 
(8) Tétreault, N.; Arsenault, E.; Heiniger, L.-P.; Soheilnia, N.; Brillet, J.; Moehl, T.; Zakeeruddin, S.; Ozin, G. A.; Grätzel, M. High-Efficiency Dye-Sensitized Solar Cell with ThreeDimensional Photoanode. Nano Letters 2011, 11, 4579-4584.

(9) Esmanski, A.; Ozin, G. A. Silicon Inverse-Opal-Based Macroporous Materials as Negative Electrodes for Lithium Ion Batteries. Adv. Funct. Mater. 2009, 19, 1999-2010.

(10) Kim, O.-H.; Kang, S. H.; Park, H.-Y.; Kim, M.; Lim, J. W.; Chung, D. Y.; Lee, M. J.; Choe, H.; Cho, Y.-H.; Sung, Y.-E. Ordered Macroporous Platinum Electrode and Enhanced Mass Transfer in Fuel Cells Using Inverse Opal Structure. Nat Comm 2013, 4, 1-9.

(11) Raccis, R.; Nikoubashman, A.; Retsch, M.; Jonas, U.; Koynov, K.; Butt, H.-J.; Likos, C. N.; Fytas, G. Confined Diffusion in Periodic Porous Nanostructures. ACS Nano 2011, 5, 46074616.

(12) Cherdhirankorn, T.; Retsch, M.; Jonas, U.; Butt, H.-J.; Koynov, K. Tracer Diffusion in Silica Inverse Opals. Langmuir 2010, 26, 10141-10146.

(13) Hatton, B.; Mishchenko, L.; Davis, S.; Sandhage, K.; Aizenberg, J. Assembly of LargeArea, Highly Ordered, Crack-Free Inverse Opal Films. Proc. Natl. Acad. Sci. U.S.A. 2010, 107, $10354-10359$.

(14) Burgess, I. B.; Mishchenko, L.; Hatton, B. D.; Kolle, M.; Lončar, M.; Aizenberg, J. Encoding Complex Wettability Patterns in Chemically Functionalized 3D Photonic Crystals. $J$. Am. Chem. Soc. 2011, 133, 12430-12432.

(15) Fenzl, C.; Hirsch, T.; Wolfbeis, O. S. Angew. Chem. Int. Ed. Photonic Crystals for Chemical Sensing and Biosensing. 2014, 53, 3318-3335. 
(16) Potyrailo, R. A.; Ghiradella, H.; Vertiatchikh, A.; Dovidenko, K.; Cournoyer, J. R.; Olson, E. Morpho Butterfly Wing Scales Demonstrate Highly Selective Vapour Response. Nature Photonics 2007, 1, 123-128.

(17) Potyrailo, R. A.; et al. Discovery of the Surface Polarity Gradient on Iridescent Morpho Butterfly Scales Reveals a Mechanism of Their Selective Vapour Response. Proc. Natl. Acad. Sci. U.S.A. 2013, 110, 15567-15572.

(18) Jung, G.-Y.; Li, Z.; Wu, W.; Chen, Y.; Olynick, D. L.; Wang, S.-Y.; Tong, W. M.; Williams, R. S. Vapor-Phase Self-Assembled Monolayer for Improved Mold Release in Nanoimprint Lithography. Langmuir 2005, 21, 1158-1161.

(19) Vogel, N.; Belisle, R. A.; Hatton, B.; Wong, T.-S.; Aizenberg, J. Transparency and Damage Tolerance of Patternable Omniphobic Lubricated Surfaces Based on Inverse Colloidal Monolayers. Nature Comm. 2013, 4, 2167.

(20) Burgess, I. B.; Koay, N.; Raymond, K. P.; Kolle, M.; Lončar, M.; Aizenberg, J. Wetting in Color: Colorimetric Differentiation of Organic Liquids with High Selectivity. ACS Nano 2012, $6,1427-1437$.

(21) Cao, L.; Hu, H.-H.; Gao, D. Design and Fabrication of Micro-textures for Inducing a Superhydrophobic Behavior on Hydrophilic Materials. Langmuir 2007, 23, 4310-4314.

(22) Tuteja, A.; Choi, W.; Ma, M.; Mabry, J. M.; Mazzella, S. A.; Rutledge, G. C.; McKinley, G. H.; Cohen, R. E. Designing Superoleophobic Surfaces. Science 2007, 318, 1618-1622.

(23) Tuteja, A.; Choi, W.; Mabry, J. M.; McKinley, G. H.; Cohen, R. E. Robust Omniphobic Surfaces. Proc. Natl. Acad.Sci. U.S.A. 2008, 105, 18200-18205. 
(24) Phillips, K. R.; Vogel, N.; Hu, Y.; Kolle, M.; Perry, C. C.; Aizenberg, J. Tunable Anisotropy in Inverse Opals and Emerging Optical Properties. Chem. Mater. 2014, 26, 16221628.

(25) Goodwin, J. W.; Hearn, J.; Ho, C. C.; Ottewill, R. H. Studies on the Preparation and Characterization of Monodisperse Polystyrene Latices: III, Preparation without added surface active agents. Colloid and Polymer Science 1974, 252, 464-471.

(26) De Gennes, P. G.; Brochard-Wyart, F.; Quéré, D. Capillarity and Wetting Phenomena: Drops, Bubbles, Pearls, Waves. Springer, 2010.

(27) Extrand, C. W.; Moon, S. I. Contact Angles on Spherical Surfaces. Langmuir 2008 24, 9470-9473.

(28) Sheng, X.; Zhang, J.; Jiang, L. Application of the Restricting Flow of Solid Edges in Fabricating Superhydrophobic Surfaces. Langmuir 2009 25, 9903-9907.

(29) Extrand, C. W.; Moon, S. I. Intrusion Pressure to Initiate Flow through Pores between Spheres. Langmuir 2012 28, 3503-3509.

(30) Park, J.-G.; Lee, S. H.; Ryu, J.-S.; Hong, Y.-K.; Kim, T.-G.; Busnaina, A. A. Interfacial and Electrokinetic Characterization of IPA Solutions Related to Semiconductor Wafer Drying and Cleaning. J. Electrochem. Soc. 2006, 153, G811-G814.

(31) Shneidman, A. V.; Burgess, I. B.; Kolle, M.; Quan, Q.; Aizenberg, J.; Lončar, M. Bioinspired tunable disorder in a 3D photonic crystal via highly controlled partial wetting and drying. Conference on Lasers and Electro-Optics 2012, Optical Society of America Technical Digest, 2012, paper QF2H.5. 


\section{TOC GRAPHIC}

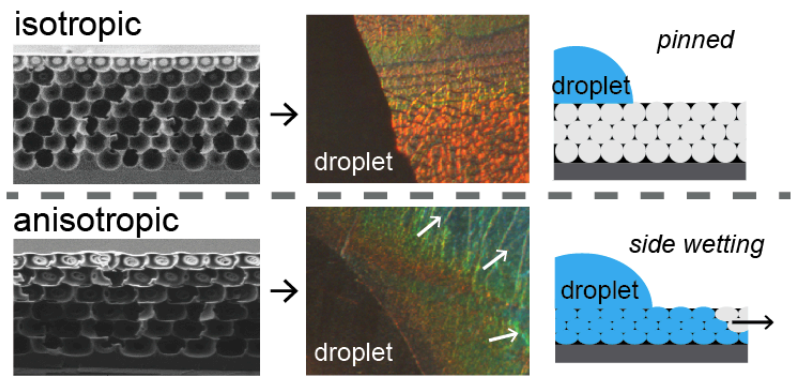

